\title{
Screening for cardiovascular risk factors and self-rated health in a community setting:
}

\author{
a cross-sectional study in Finland
}

\begin{abstract}
Background

Self-rated health is an independent predictor of mortality. However, general health checks in populations unselected for disease or risk factors have not been shown to reduce mortality or morbidity.
\end{abstract}

\section{Aim}

To describe new comorbidities and cardiovascular risk factors in apparently healthy people and to relate this to their selfrated health.

\section{Design and setting}

A targeted screening programme identified 462 middle-aged people with cardiovascular risk factors without previously diagnosed chronic disease in a Finnish community in 2005-2006.

\section{Method}

Home blood pressure monitoring, oral glucose tolerance test, estimated glomerular filtration rate, and ankle brachial index were used to detect previously undiagnosed conditions. The Short-Form Health Survey and Beck's Depression Inventory were completed by participants before the diagnostic tests were performed.

\section{Results}

The prevalence of previously undiagnosed disease was: hypertension 113/462 (24\% [95\% confidence interval $\{\mathrm{Cl}\}=21 \%$ to $29 \%]$. diabetes $19 / 462$ (4\% [95\% Cl $=2 \%$ to $6 \%]$ ), renal insufficiency $23 / 462$ (5\% [95\% Cl $=3 \%$ to $7 \%]$ ), and peripheral arterial disease 17/462 (4\% [95\% $\mathrm{Cl}=2 \%$ to $5 \%]$ ]. Of the 139 participants who regarded their health as 'fair-poor', 60 (43\%) had a previously undetected condition affecting their vasculature.

\section{Conclusion}

Out of the screen-detected apparently healthy cardiovascular risk subjects, one in three had undiagnosed hypertension, diabetes, peripheral arterial disease, or renal insufficiency. Those individuals experiencing ill health tended to be at high risk of cardiovascular problems.

\section{Keywords}

cardiovascular disease; community health;

health promotion; prevention; primary care; risk factors; screening

\section{INTRODUCTION}

A Cochrane Review carried out in 2012 showed that general health checks do not reduce mortality or morbidity, although they lead to more diagnoses and medical treatment. ${ }^{1}$ This finding has shaken the concept of primary prevention, which is performed in apparently healthy individuals to postpone the onset of symptoms so they can live longer and healthier lives. The prevention of cardiovascular disease particularly has been an important goal in general practice, since cardiovascular disease remains the major cause of noncommunicable diseases in the world. ${ }^{2}$

However, there are strong arguments in favour of strategies promoting primary prevention programmes. In the UK, it has been estimated that reductions in total cholesterol, mean blood pressure, and prevalence of smoking in apparently healthy people achieved a fourfold larger reduction in coronary heart disease deaths between 1981 and 2000 than risk factor reduction in patients with coronary heart disease (secondary prevention). ${ }^{3}$ In Finland, coronary heart disease mortality rates decreased by 63\% between 1982 and 1997, which can be attributed largely to reductions in population risk factors and also to improvements of medical treatments. ${ }^{4}$

Self-rated health is known to be an independent predictor of mortality. ${ }^{5,6}$

PE Korhonen, MD, PhD, specialist in family medicine and internal medicine, Satakunta Hospital District, Pori; Central Satakunta Health Federation of Municipalities, Harjavalta; Institute of Clinical Medicine, Family Medicine, University of Turku, Turku, Finland. H Kautiainen, BA, statistician, Unit of Primary Health Care, Helsinki University Central Hospital; Department of General Practice, University of Helsinki, Helsinki, Finland. P Mäntyselkä, MD, PhD, specialist in family medicine, Primary Health Care Unit, Institute of Public Health and Clinical Nutrition, School of Medicine, University of Eastern Finland; Primary Health Care Unit, Kuopio University
However, information about patients wellbeing from their own point of view is too often missing in health care. The aim of the present study was to describe new comorbidities and cardiovascular risk factors in apparently healthy people who are at increased risk of cardiovascular disease, and to relate this to their self-rated health.

\section{METHOD}

\section{Participants}

The participants of the present study were drawn from a targeted screening programme for cardiovascular risk factors, which was carried out in the rural town of Harjavalta in southwestern Finland in 2005-2006.

A risk factor survey was posted to all inhabitants aged $45-70$ years living at home ( $n=2856$ ). Participation and all of the tests included were free of charge for the subjects. Of the 2085 (73\%) responders, those found to have at least one cardiovascular risk factor ( $n=1756)$ were invited for an enrolment examination. Risk factors taken into account were a waist circumference of at least $80 \mathrm{~cm}$ in females and $94 \mathrm{~cm}$ in males, a latest measured blood pressure $\geq 140 \mathrm{mmHg}$ systolic (SBP) or $\geq 90 \mathrm{mmHg}$ diastolic (DBP), a history of gestational hypertension or diabetes, a family history of premature cardiovascular disease, and a score of $\geq 12$ in the Finnish Diabetes Risk
Hospital, Kuopio, Finland

\section{Address for correspondence}

Päivi Korhonen, Jokikatu 3, 29200 Harjavalta, Finland.

E-mail: paivi.e.korhonenafimnet.fi

Submitted: 6 April 2014; Editor's response: 1 May 2014; final acceptance: 13 May 2014. (c)British Journal of General Practice This is the full-length article (published online 29 Sep 2014) of an abridged version published in print. Cite this article as: $\mathbf{B r} \mathbf{J}$ Gen Pract 2014; DOI: 10.3399/bjgp14X681769 


\section{How this fits in}

Self-rated health is known to be an independent predictor of mortality, but general health checks in populations unselected for disease or risk factors have not been shown to reduce mortality. This study used a targeted screening programme and identified apparently healthy middle-aged people with cardiovascular risk factors in the community. One in three of them were found to have undiagnosed hypertension, diabetes, peripheral arterial disease, or renal insufficiency. Moreover, many subjects with undiagnosed comorbidities felt general ill health. Self-rated health may therefore be important to include in screening programmes.

Score. ${ }^{7}$ Of the risk subjects, 1469 out of 1756 $(84 \%)$ attended the enrolment examination at the community health centre. Trained public health nurses performed the enrolment examination, which included a physical examination, laboratory tests, and a medical history.

Information about chronic comorbidities was gathered from questionnaires, medical records, medications, and physical examination. For the analyses described here, patients with previously diagnosed chronic diseases (cardiovascular disease, renal disease, diabetes, hypertension, cancer, neurological disorders, pulmonary disorders, musculoskeletal, or connective tissue disorders) were excluded, yielding a cohort of 462 apparently healthy individuals.

All participants provided written informed consent for the project and subsequent medical research.

\section{Measurements and definitions}

A 2-hour oral glucose tolerance test was performed, and type 2 diabetes was diagnosed according to the World Health Organization's 2006 criteria. ${ }^{8}$ Hypertension was diagnosed if the mean SBP taken by the nurse was $\geq 140 \mathrm{mmHg}$ or the mean DBP was $\geq 90 \mathrm{mmHg}$, and if the mean home blood pressure monitoring was $\geq 135 \mathrm{mmHg}$ systolic or $\geq 85 \mathrm{mmHg}$ diastolic.

Height and weight were measured by the nurse. Body mass index was calculated as weight ( $\mathrm{kg}$ ) divided by height squared $\left(\mathrm{m}^{2}\right)$.

Subclinical organ damage was assessed by measuring the ankle brachial index and estimated glomerular filtration rate. The ankle brachial index was calculated by dividing the lower ankle SBP by the higher brachial SBP. ${ }^{10}$ An ankle brachial index of $\leq 0.90$ in either leg was considered to indicate peripheral arterial disease. The estimated glomerular filtration rate was determined using the four-variable Modification of Diet in Renal Disease Study equation. ${ }^{11}$ Estimated glomerular filtration rate levels below $60 \mathrm{ml} / \mathrm{min} / 1.73 \mathrm{~m}^{2}$ were reported as renal insufficiency.

\section{Questionnaires}

Participants completed self-administrated questionnaires at the clinic before the enrolment examination was performed. These measured sociodemographic factors, occupational status, physical activity level, smoking status, and health-related quality of life using the Short-Form Health Survey (SF-36), and Beck's Depression Inventory (BDI). 12,13 The first question of the SF-36 was used to assess self-rated health in categories of good-excellent; and fair-poor. Participants with a BDI score of $\geq 10$ were regarded as having depressive symptoms. ${ }^{14}$

Leisure-time physical activity (LTPA) was classified as: high - LTPA of at least 30 minutes at a time for at least six times a week; moderate - LTPA of at least 30 minutes at a time from four to five times a week; and low - LTPA of at least 30 minutes at a time three times a week or less.

\section{Statistical analyses}

Data are presented as means with standard deviations or as counts with percentages. The most important results are given with 95\% confidence intervals (Cls). Statistical comparison between groups in measures with binary distribution was made by $\chi^{2}$ or Fisher's exact test, when appropriate. The independent samples $t$-test was used for continuous variables, except for biochemical variables, which were analysed using the bootstrapped type $t$-test because of their skewed distributions.

\section{RESULTS}

Of the 462 apparently healthy cardiovascular risk subjects (mean age $57 \pm 7$ years, $50 \%$ female), the prevalence of previously undiagnosed diseases were: hypertension 113 out of $462(24 \%$ [ $95 \% \mathrm{Cl}=21 \%$ to $29 \%]]$ ), diabetes 19 out of $462(4 \%[95 \% \mathrm{Cl}=2 \%$ to $6 \%]$ ), renal insufficiency 23 out of 462 (5\% $[95 \% \mathrm{Cl}=3 \%$ to $7 \%]$, and peripheral arterial disease 17 out of 462 (4\% [95\% Cl $=2 \%$ to $5 \%]$. Eighteen subjects had at least two of these conditions. Thus, 154 out of 462 (33\%) of the apparently healthy cardiovascular risk subjects were not truly healthy.

Table 1 shows the characteristics of the participants. Compared with participants 
Table 1. Characteristics of the participants

\begin{tabular}{|c|c|c|c|}
\hline & \multicolumn{2}{|c|}{ Self-rated health } & \multirow[b]{2}{*}{$P$-value } \\
\hline & $\begin{array}{c}\text { Fair-poor } \\
n=139\end{array}$ & $\begin{array}{c}\text { Good-excellent } \\
\qquad n=323\end{array}$ & \\
\hline \multicolumn{4}{|l|}{ Demographic } \\
\hline Female, $n(\%)$ & $68(49)$ & $162(50)$ & 0.81 \\
\hline Age in years, mean (SD) & $59(7)$ & $57(7)$ & 0.005 \\
\hline Blue-collar workers, $n(\%)$ & 113 (87) & $215(70)$ & $<0.001$ \\
\hline LTPA low, $n(\%)$ & $34(26)$ & 40 (13) & $<0.001$ \\
\hline Smokers, $n(\%)$ & $34(24)$ & $58(18)$ & 0.11 \\
\hline \multicolumn{4}{|l|}{ Clinical } \\
\hline Systolic blood pressure, mmHg, mean (SD) & $148(19)$ & $146(17)$ & 0.37 \\
\hline Diastolic blood pressure, mmHg, mean (SD) & $88(9)$ & $88(8)$ & 0.76 \\
\hline eGFR, ml/min, mean (SD) & $84(17)$ & $78(16)$ & $<0.001$ \\
\hline Body mass index, kg/m², mean (SD) & $29.5(5.3)$ & $28.1(4.2)$ & 0.002 \\
\hline \multicolumn{4}{|l|}{ Waist, cm, mean (SD) } \\
\hline Male & 102 (13) & $99(9)$ & 0.023 \\
\hline Female & 94 (13) & $92(11)$ & 0.18 \\
\hline Obesity, body mass index $\geq 30 \mathrm{~kg} / \mathrm{m}^{2}, n[\%]$ & $56(40)$ & $90(28)$ & 0.008 \\
\hline Metabolic syndrome, $n(\%)$ & $67(48)$ & $162(50)$ & 0.70 \\
\hline Beck's Depression Inventory $\geq 10, n(\%)$ & $35(26)$ & $28(9)$ & $<0.001$ \\
\hline \multicolumn{4}{|l|}{ Biochemical } \\
\hline Total cholesterol, mmol/l, mean (SD) & 5.32 (1.03) & $5.35(0.95)$ & 0.76 \\
\hline HDL-cholesterol, mmol/l, mean (SD) & $1.49(0.44)$ & $1.47(0.39)$ & 0.71 \\
\hline LDL-cholesterol, mmol/l, mean (SD) & $3.21(0.87)$ & $3.28(0.87)$ & 0.46 \\
\hline Triglycerides, mmol/l, mean (SD) & $1.39(0.64)$ & $1.36(0.38)$ & 0.67 \\
\hline Fasting plasma glucose, mmol/l, mean (SD) & $5.61(0.98)$ & $5.61(0.78)$ & 0.99 \\
\hline 2-hour plasma glucose, mmol/l, mean (SD) & $7.70(2.66)$ & $7.21(2.17)$ & 0.037 \\
\hline
\end{tabular}

who felt their health to be good or excellent, those who felt ill health were slightly older, more often blue-collar workers llow socioeconomic status), had lower LTPA, higher BMI, wider waist circumference if male, and more often depressive symptoms. Of the biochemical factors, only 2-hour plasma glucose values were significantly higher in those who felt ill health.

Of the 323 participants who regarded their health as being good-excellent, 94 $(29 \%)$ were not truly healthy, while 60 out of 139 (43\%) whose self-rated health was fairpoor were not healthy $(P=0.003)$. Among those who felt ill health, the age- and sexadjusted odds ratio (OR) for having one of the screened diseases was $1.76195 \% \mathrm{Cl}$ $=1.16$ to $2.67, P=0.008)$, for hypertension OR $1.43(95 \% \mathrm{Cl}=0.91$ to $2.24, P=0.12)$, for type 2 diabetes OR 2.13 (95\% Cl 0.84 to 5.43 , $P=0.11)$, for peripheral arterial disease OR $1.88(95 \% \mathrm{Cl}=0.70$ to $5.04, P=0.21)$, and for renal insufficiency OR $1.53(95 \% \mathrm{Cl}=0.63$ to 3.69, $P=0.34$ ).

\section{DISCUSSION}

Summary

Of the screen-detected apparently healthy cardiovascular risk subjects, one in three were not truly healthy when diagnostic tests easily available to a GP were used to detect undiagnosed conditions affecting the vasculature. These not-healthy individuals (defined as having previously undetected hypertension, type 2 diabetes, peripheral arterial disease, or renal insufficiencyl also more often had a sense of general ill health than participants who were truly healthy in regard to these conditions.

\section{Strengths and limitations}

The strengths of this study are that the data come from a community-based representative sample of the middle-aged Finnish population, bias regarding previously detected comorbidities was excluded, and the health of the study participants was assessed from their own point of view and that of a medical professional. The major limitation of this study is the cross-sectional design, which prevents the study from drawing conclusions regarding any causal associations.

\section{Comparison with existing literature}

The 2012 Cochrane analysis only used 
randomised controlled trials, which may not be the best design to study effectiveness of prevention programmes. ${ }^{1}$ Most of the studies included in the Cochrane Review were conducted more than 20 years ago, before statins were invented and when modern antihypertensive agents were not in general use. Moreover, most studies included people at low overall cardiovascular risk; many did not specifically include cardiovascular disease risk and were not conducted in primary care.

The screening programme in the present study was targeted at cardiovascular risk subjects among whom the likelihood of having disease is higher than in the general population. It can therefore be considered reasonable to detect further risk and early signs of cardiovascular disease. The programme also included a patient-centred approach by assessing health-related quality of life before the clinical tests were obtained. A meta-analysis of prospective cohort studies showed that poor self-rated health is strongly associated with mortality, even after adjustment for known comorbidities. ${ }^{5}$ However, longitudinal follow-up is needed to demonstrate the failure or success of this screening programme.

\section{Implications for clinical practice}

The results suggest that in people at increased cardiovascular risk, hypertension, type 2 diabetes, peripheral arterial disease, or renal insufficiency are frequent. Although these diseases have often been regarded as symptomless, participants more often had a sense of generally poorer health than the participants who were truly healthy with regard to these conditions. The value of self-rated health as an independent predictor of mortality, 5 and the results of the present study suggest that this may be due to undiagnosed comorbidities. Selfrated health may therefore be important to include in screening programmes.

\section{Funding}

Satakunta Hospital District provided financial support for the statistical analysis.

\section{Ethical approval}

The study protocol and consent forms were reviewed and approved by the Ethics Committee of Satakunta Hospital District.

\section{Provenance}

Freely submitted; externally peer reviewed.

\section{Competing interests}

The authors have declared no competing interests.

\section{Acknowledgements}

The study was conducted in Harjavalta Health Center, at the Central Satakunta Health Federation of Municipalities.

\section{Discuss this article}

Contribute and read comments about this article: www.bjgp.org/letters 


\section{REFERENCES}

1. Krogsbøll LT, Jørgensen KJ, Grønhøj L, Gøtzsche PC. General health checks in adults for reducing morbidity and mortality from disease: Cochrane systematic review and meta-analysis. BMJ 2012; 345: e7191.

2. World Health Organization. Global status report on noncommunicable diseases: 2010. Geneva: WHO, 2011

3. Unal B, Critchley JA, Capewell S. Modelling the decline in coronary heart disease deaths in England and Wales, 1981-2000: comparing contributions from primary prevention and secondary prevention. BMJ 2005; 331: 614-619.

4. Vartiainen E, Laatikainen T, Salomaa V, et al. Effects of risk factors and treatments to the decline of coronary heart disease mortality in Finland. Finnish Med J 2004; 37: 3373-3377.

5. DeSalvo KB, Bloser N, Reynolds K, et al. Mortality prediction with a single general self-rated health question. A meta-analysis. J Gen Intern Med 2006; 21 267-275.

6. Idler EL, Benyamini Y. Self-rated health and mortality: a review of twenty-seven community studies. J Health Soc Behav 1997; 38: 21-37.

7. Lindström J, Tuomilehto J. The diabetes risk score: a practical tool to predict type 2 diabetes risk. Diabetes Care 2003; 26: 725-731.
8. World Health Organization. Definition and diagnosis of diabetes mellitus and intermediate hyperglycemia. Report of a WHO/IDF consultation. http://www.who. int/diabetes/publications/Definition\%20and\%20diagnosis\%20of\%20diabetes_ new.pdf laccessed 19 Aug 2014).

9. Parati G, Stergiou GS, Asmar R, et al. European Society of Hypertension guidelines for blood pressure monitoring at home: a summary report of the Second International Consensus Conference on Home Blood Pressure Monitoring. J Hypertens 2006; 26: 1505-1526.

10. Espinola-Klein C, Rupprecht HJ, Bickel C, et al. Different calculations of ankle brachial index and their impact on cardiovascular risk prediction. Circulation 2008; 118: 961-967.

11. Levey AS, Coresh J, Greene T, et al. Expressing the Modification of Diet in Renal Disease Study equation for estimating glomerular filtration rate with standardized serum creatinine values. Clin Chem 2007; 53: 766-772.

12. Hays RD, Morales LS. The RAND-36 measure of health-related quality of life. Ann Med 2001; 33: 350-357.

13. Beck AT, Wand $\mathrm{CH}$, Mendelson $\mathrm{M}$, et al. An inventory for measuring depression. Arch Gen Psychiatry 1961; 4: 561-571.

14. Koponen H, Jokelainen J, Keinänen-Kiukaanniemi S, Vanhala M. Depressive symptoms and 10-year risk for cardiovascular morbidity and mortality. World J Biol Psychiatry 2010; 11: 834-839. 